OPEN ACCESS

Edited by:

Saumya Panda,

KPC Medical College and

Hospital, India

Reviewed by:

Efterpi Zafiriou,

University of Thessaly, Greece

Guillaume Luxardi,

University of California, Davis,

United States

*Correspondence:

Rahul Mahajan

drrahulpgi@yahoo.com

Specialty section:

This article was submitted to

Dermatology,

a section of the journal

Frontiers in Medicine

Received: 20 May 2021

Accepted: 28 January 2022

Published: 21 February 2022

Citation:

Thakur V and Mahajan R (2022) Novel Therapeutic Target(s) for Psoriatic Disease. Front. Med. 9:712313. doi: 10.3389/fmed.2022.712313

\section{Novel Therapeutic Target(s) for Psoriatic Disease}

\author{
Vishal Thakur and Rahul Mahajan* \\ Department of Dermatology, Venereology, and Leprology, Postgraduate Institute of Medical Education and Research, \\ Chandigarh, India
}

Psoriasis and psoriatic arthritis, together known as psoriatic disease, is highly prevalent chronic relapsing inflammatory disease affecting skin, joints or both and is associated with several comorbidities such as cardiovascular, metabolic, psychiatric, renal disease etc. The etiopathogenesis of psoriasis is complex and mainly driven by aberrant immune response owing to the genetic susceptibility and various environmental factors such as trauma, infections and drugs. Recent advances in understanding molecular and cellular pathways have identified tumor necrosis factor- $\alpha$ (TNF- $\alpha$ ), interleukin-17 (IL-17), IL-23, IL-22 as major contributors in psoriasis pathogenesis. Advances in the knowledge of pathophysiology, the interaction of autoinflammation and clinical phenotypes have led to the development of highly effective targeted therapeutic agents which include TNF- $\alpha$, IL-17, IL-23, IL-1 $\alpha / \beta$ or IL-36 inhibitors or receptor blockers, small molecule drugs like phosphodiesterase-4 inhibitors (apremilast), Janus kinase (JAK) inhibitors, retinoic acid receptor-related orphan receptor $\gamma t(R O R \gamma t)$ inhibitors. These novel drugs have promised the potential of improved disease control. In recent years, the transition from biologics to biosimilars especially with TNF- $\alpha$ inhibitors had significant impact on decreasing health care cost and increasing therapeutic options to the patients. However, selection of right treatment for an individual patient still remains challenging. Moreover, interplay between different epigenetic mechanisms such as the DNA methylation, chromatin modifications and noncoding RNA regulation has recently been started to be deciphered. Enzymes inhibitors involved in epigenetic pathways such as DNA methyltransferases and histone deacetylases demonstrated to restore normal epigenetic patterns in clinical settings and have provided the potential as novel therapeutic targets for psoriasis. In this review, we will discuss novel biologic agents and newer therapeutic approaches in treatment of psoriatic disease.

Keywords: psoriasis, psoriatic arthritis, infliximab monotherapy, autoimmune hepatitis, treatment, biologics and biosimilars, small molecule

\section{INTRODUCTION}

Psoriatic disease is a chronic relapsing inflammatory condition affecting $\sim 2-3 \%$ of population $(1,2)$. Psoriatic disease consists of psoriasis vulgaris affecting skin and psoriatic arthritis affecting joints. Psoriasis affects patients' quality of life significantly and have tremendous psychosocial burden among patients (3). The immunopathogenesis of psoriasis is complex primarily driven by an aberrant immune response further modified by an interplay between genetic susceptibility 
and environmental factors. The inflammatory events lead to systemic inflammation resulting in cardiovascular, metabolic and renal disease and increased morbidity (4). In last few years, advances in understanding molecular and cellular pathways have identified tumor necrosis factor- $\alpha$ (TNF- $\alpha$ ), interleukin-17 (IL17), IL-23, IL-22 as major contributors in psoriasis pathogenesis (5). This has led to the development of highly effective targeted therapeutic agents which include TNF- $\alpha$, IL-17, IL-23, IL-1 $\alpha / \beta$ or IL-36 inhibitors or receptor blockers, small molecule drugs like phosphodiesterase-4 inhibitors (apremilast), Janus kinase (JAK) inhibitors, retinoic acid receptor-related orphan receptor$\gamma \mathrm{T}(\mathrm{ROR} \gamma \mathrm{T})$ inhibitors (5). Figure 1 shows the pathogenesis and various therapeutic targets in psoriatic disease. These novel drugs have promised the potential of improved disease control. In this review, we will discuss novel therapeutic targets in the management of psoriatic disease.

\section{JAK INHIBITORS}

The Janus Kinase-Signal Transducer and Activator of Transcription (JAK-STAT) pathway plays an important role in intracellular signaling in various physiological and pathological processes in inflammatory disorders including psoriasis. Cytokines implicated in psoriasis pathogenesis mainly IL-17, IL-23, TNF- $\alpha$, IL-1, IL-22, IFN- $\alpha$ and IFN- $\gamma$ are linked to JAK-STAT pathway $(6,7)$. Upon interaction of various cytokines with their respective receptor, activation of JAK leads to phosphorylation of STAT proteins and nuclear translocation resulting in gene expression (8). In psoriasis, increased expression and upregulation of STAT1 and STAT3 have been demonstrated in the lesional skin $(9,10)$. STAT1 and STAT3 are involved in the activation of dendritic cells and differentiation of Th1 and Th17 cells $(9,10)$. STAT3 also leads to the keratinocyte proliferation mediated through IL-19, IL-36 and IL-22 (11). IFN- $\gamma$ secreted from keratinocytes leads to the migration of inflammatory cells from the lymphoid tissue to the skin (10).

Various JAK inhibitors have been used in psoriatic disease with good efficacy, of which Tofacitinib, an oral JAK1/3 inhibitor, has been extensively studied in phase II and III trials (6). In phase III studies, a significant proportion of patients achieved PASI75 at weeks 12 or 16 showing greater efficacy with higher doses i.e., $10 \mathrm{mg}$ twice daily (12). Studies evaluating the efficacy after treatment withdrawal also showed higher efficacy as compared to placebo (13). In another study, 74.1 and $79.4 \%$ of patients receiving tofacitinib $5 \mathrm{mg}$ twice daily and $10 \mathrm{mg}$ twice daily respectively, maintained the response at 52 -weeks (14). Tofacitinib has shown significantly better efficacy and safety in psoriatic arthritis as compared to placebo $(15,16)$. A topical formulation of tofacitinib has also been developed and used in plaque psoriasis with modest efficacy (17). Common adverse effects include cytopenia and infections $(6,18)$. Safety concerns especially dose-dependent (i.e., $10 \mathrm{mg}$ twice daily) risk of herpes zoster, higher chances of infections, gastric perforation and thromboembolic events has been raised $(6,18)$, although longterm studies with larger samples are needed. Due to these safety concerns, tofacitinib was not approved for psoriasis by FDA, however it is approved for use in psoriatic arthritis (6).

Baricitinib, an oral highly selective JAK1 and JAK2 inhibitor has also been studied in patients with moderate-to-severe psoriasis in Phase II trials and has shown better efficacy as compared to placebo at doses $8 \mathrm{mg}$ and $10 \mathrm{mg}$ (19). Adverse effects included anemia, cytopenia and increase in creatinine levels (6). Similar safety concerns have been raised with baricitinib, thus, it is approved for use in rheumatoid arthritis only.

Ruxolitinib, another JAK1 and JAK2 inhibitor, has been developed as topical cream and studies in psoriasis showed a better efficacy and safety profile compared to vehicle and Non-inferior to calcipotriol-betamethasone combination (17). Other JAK1/2 inhibitors such as itacitinib (20), abrocitinib (21), solcitinib (22) and filgotinib (23) have shown efficacy in phase II trials in psoriasis and psoriatic arthritis. Peficitinib, an oral panJAK inhibitor with JAK3 selectivity, showed a good efficacy in psoriasis in phase IIa trial with no major adverse events (24).

\section{IL-23}

IL-23, a cytokine of IL-12 family, consists of two subunits: p19 (unique for IL-23) and p40 that is common with IL-12 (25). IL-23 is mainly produce by dendritic cells and macrophages $(26,27)$. Initially, antibodies targeting $\mathrm{p} 40$ subunit of IL-12 were found effective in psoriasis as these neutralized IL-23 also (27). Later on, increased expression of p19 and p40 was found in psoriatic lesions while p35 that is specific to IL-12 was normal which suggested that IL-23 not IL-12 is an important cytokine involved in the psoriasis pathogenesis (26). IL-23 binds to its heterodimeric receptor leading to the activation of Janus kinases (Jak) and further activation of STAT3 (28). IL-23 leads to the production of cytokines from Th-17 cells i.e., IL-17, a major cytokine implicated in the pathogenesis of psoriasis (28). This led to development of anti-IL23 biologics in the therapeutics of psoriatic disease. As these agents target upstream cytokine involved in the psoriasis pathogenesis, dosing interval of longer duration is an advantage as compared to the downstream cytokines such as IL-17 and TNF- $\alpha$ (29). Currently, ustekinumab, guselkumab, tildrakizumab, and risankizumab are FDA approved for psoriasis vulgaris and only ustekinumab and guselkumab have been approved by the FDA for psoriatic arthritis (29). IL-23 inhibitors have shown superior efficacy to conventional agents and TNF- $\alpha$ inhibitors. A network metanalysis found guselkumab and risankizumab more effective than tildrakizumab (30). The IL-23 inhibitors have been found to be more effective in maintaining remission as compared to other drugs even after drug discontinuation. In PHOENIX 1 trial of ustekinumab, median time to loss of PASI-50 was $\sim 22$ weeks from the last dose of drug (31). Similar results have been observed with other IL-23 inhibitors including guselkumab, tildrakizumab and risankizumab, showing sustained improvement in disease after drug discontinuation (32-34). The efficacy of IL-23 inhibitors especially ustekinumab and guselkumab in psoriatic arthritis was also found significantly high as compared to placebo 


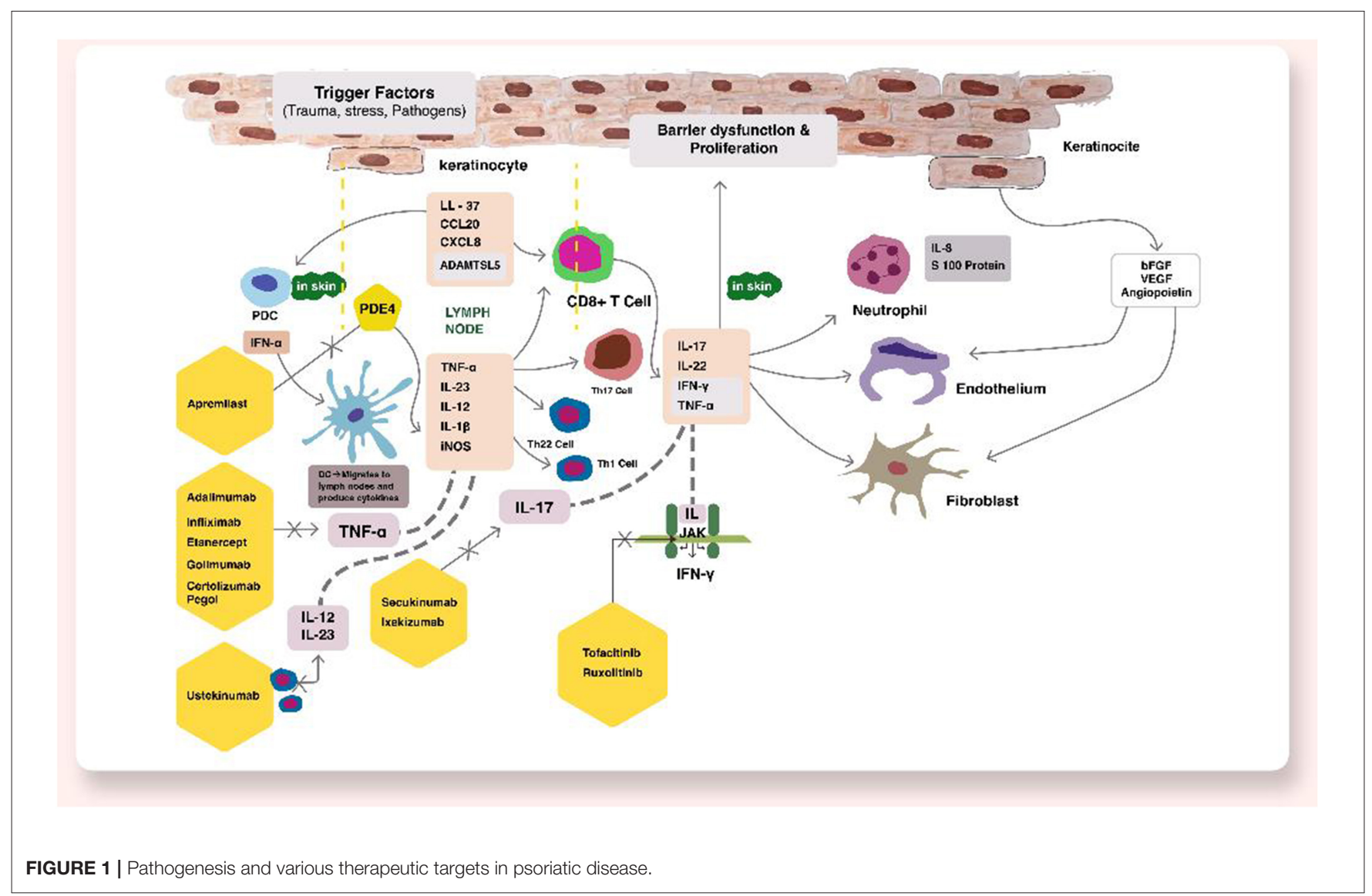

(35-37). However, more studies evaluating efficacy of these agents and comparison with other drugs such as TNF- $\alpha$ are required. Common adverse effects of IL-23 inhibitors include upper respiratory infections, nasopharyngitis, and headache (29). Other advents events include serious infections, major adverse cardiovascular events and malignancy, however, the rates observed were comparable to seen in general population of psoriasis patients (29). A long-term data on the safety of these novel drugs is thus warranted.

\section{IL-36}

IL-36 (member of IL-1 family) binds to its receptor and leads to the activation of NF- $\mathrm{B}$ and MAPKs pathways through MyD88/IRAK complex (38). Expression of IL-36y have been found to be significantly upregulated in the serum and skin samples of psoriasis patients (39). Furthermore, loss of function mutation in IL-36Ra gene has been found in a severe variant of generalized pustular psoriasis (GPP) $(40,41)$. Studies in mouse model have observed psoriasis like epidermal changes, inflammatory cell infiltrate and gene dysregulation after IL-36 administration which was not seen when Pre-treatment with an IL-36 antagonist was administered (42). This supports a direct role of IL-36 in psoriasis pathogenesis and attenuating this signaling pathway may be an effective alternative approach to the already approved small molecules such as apremilast or other biologics. Moreover, studies have shown that individuals with loss of function mutation in IL-36Ra gene have normal immune function suggesting that targeting this cytokine may not lead to adverse events associated with immune dysregulation and may have a good safety profile (43). Recently, an oral small molecule inhibitor of IL-36, A-552 was shown to inhibit IL$36 \gamma$ and production of other cytokines induced by IL-36 $\gamma$ in human and mouse cells (44). Monoclonal antibody against IL36R, spesolimab has shown efficacy in a Phase I study, and phase II and III studies of spesolimab in GPP are currently undergoing $(45,46)$. Thus, anti-IL-36 agents may have a robust potential in therapeutics of psoriasis and further research evaluating their efficacy and safety is needed. Table 1 summarizes the studies of JAK inhibitors, IL-23 and IL-36 inhibitors in psoriasis and psoriatic arthritis.

\section{IL-1}

IL-1, a proinflammatory cytokine, comprise of IL- $1 \alpha$ and IL-1 $\beta$. Both these cytokines has been implicated in the pathogenesis of psoriasis (46). Increased expression of IL-1 $\beta$ has been found in the psoriatic skin and correlated with disease severity (47). Furthermore, IL-1 $\beta$ has been shown to induce Th17 cells and stimulate keratinocytes to secrete chemokines such as CCL20 (47). IL-1 $\beta$ production is also regulated by NLRP3 inflammasome as these inflammasomes cleave procaspases into caspases leading 
TABLE 1 | Summary of various trials of JAK inhibitors, IL-23, IL-12/23 and IL-36 inhibitors.

\begin{tabular}{|c|c|c|c|c|c|c|c|}
\hline Drug & Study/year & Setting/Dose & $\begin{array}{l}\text { Number of } \\
\text { patients }\end{array}$ & Response & Adverse effects & Conclusion & Phase \\
\hline \multirow[t]{5}{*}{$\begin{array}{l}\text { JAK inhibitors } \\
\text { Tofacitinib }\end{array}$} & $\begin{array}{l}\text { Papp et al. (87)/ } \\
2012\end{array}$ & $\begin{array}{l}\text { Psoriasis vulgaris-Tofacitinib } \\
2 \mathrm{mg} \text { twice daily vs. } 5 \mathrm{mg} \text { twice } \\
\text { daily vs. } 15 \mathrm{mg} \text { twice daily vs. } \\
\text { placebo }\end{array}$ & $\begin{array}{l}\text { Tofacitinib } \\
2 \text { mg- } 49 ; 5 \\
\text { mg-49; } 15 \\
\text { mg-49; } \\
\text { placebo-50 }\end{array}$ & $\begin{array}{l}\text { At week } 12 \text {, higher proportion of } \\
\text { patients achieved PASI } 75 \text { in all } \\
\text { tofacitinib groups: } 25 \cdot 0 \% \\
(2 \mathrm{mg}), 40 \cdot 8 \%(5 \mathrm{mg}) \text { and } 66 \cdot 7 \% \\
(15 \mathrm{mg}) \text { compared with placebo } \\
(2 \cdot 0 \%) \text {. }\end{array}$ & $\begin{array}{l}\text { Infections and } \\
\text { infestations, }\end{array}$ & $\begin{array}{l}\text { Oral tofacitinib results in } \\
\text { significant clinical } \\
\text { improvement in patients with } \\
\text { moderate-to-severe plaque } \\
\text { psoriasis. }\end{array}$ & Phase 2b \\
\hline & $\begin{array}{l}\text { Bisonette et al. } \\
(13) / 2015\end{array}$ & $\begin{array}{l}\text { Moderate-to-severe plaque } \\
\text { psoriasis-tofacitinib } 5 \text { mg or } \\
10 \text { mg twice daily for } 24 \text { weeks. } \\
\text { The patients achieving both } \\
\text { PASI } 5 \text { and Physician's Global } \\
\text { Assessment of "clear" or } \\
\text { "almost clear" received a } \\
\text { placebo or the previous dose. } \\
\text { At relapse (>50\% reduction in } \\
\text { the PASI improvement during } \\
\text { initial treatment) or week } 40 \text {, the } \\
\text { patients received the initial } \\
\text { dose. }\end{array}$ & $\begin{array}{l}\text { Tofacitinib } \\
5 \text { mg-331; } 10 \\
\text { mg-335 }\end{array}$ & $\begin{array}{l}33.5 \% \text { and } 55.2 \% \text { achieved } \\
\text { both PASI } 75 \text { and PGA } \\
\text { responses in tofacitinib } 5 \text { and } \\
10 \text { mg twice daily group, } \\
\text { respectively. }\end{array}$ & $\begin{array}{l}\text { Elevations in } \\
\text { low-density } \\
\text { lipoprotein- } \\
\text { cholesterol } \\
\text { levels }\end{array}$ & $\begin{array}{l}\text { Patients who received } \\
\text { continuous treatment } \\
\text { maintained a response more } \\
\text { effectively than placebo. } \\
\text { Patients who relapsed, } 60 \% \\
\text { reattained a response with } \\
\text { tofacitinib. }\end{array}$ & Phase 3 \\
\hline & $\begin{array}{l}\text { Bachelez et al. (88) } \\
\text { / } 2015\end{array}$ & $\begin{array}{l}\text { Moderate-to-severe plaque } \\
\text { psoriasis-Tofacitinib } 5 \mathrm{mg} \text { twice } \\
\text { daily vs. } 10 \mathrm{mg} \text { twice daily vs. } \\
\text { Etanercept } 50 \mathrm{mg} \text { twice weekly } \\
\text { vs. placebo }\end{array}$ & $\begin{array}{l}\text { Tofacitinib } \\
5 \text { mg-330; } 10 \\
\text { mg-332; } \\
\text { Etanercept- 336; } \\
\text { placebo- } 108\end{array}$ & $\begin{array}{l}\text { At week 12, PASI } 75-39.5 \% \text { in } \\
\text { tofacitinib } 5 \mathrm{mg} \text { group, } 63.6 \% \text { in } \\
\text { tofacitinib } 10 \mathrm{mg} \text { group, } 58.8 \% \\
\text { in the etanercept group, and } \\
5.6 \% \text { in the placebo group. }\end{array}$ & $\begin{array}{l}\text { Serious adverse } \\
\text { events-2\% in } \\
\text { tofacitinib } 5 \mathrm{mg} \\
\text { group, } 2 \% \text { in } \\
\text { tofacitinib } 10 \mathrm{mg} \\
\text { group, } 2 \% \text { in } \\
\text { etanercept group, } \\
\text { and } 2 \% \text { in placebo } \\
\text { group. }\end{array}$ & $\begin{array}{l}\text { Tofacitinib } 10 \mathrm{mg} \text { twice daily } \\
\text { was Non-inferior to } \\
\text { etanercept and was superior } \\
\text { to placebo, but } 5 \mathrm{mg} \text { twice } \\
\text { daily did not show } \\
\text { Non-inferiority to etanercept. }\end{array}$ & $\begin{array}{l}\text { Phase 3, } \\
\text { randomized, } \\
\text { multicentre, } \\
\text { placebo- } \\
\text { controlled, } \\
\text { 12-week, } \\
\text { Non-inferiority trial. }\end{array}$ \\
\hline & $\begin{array}{l}\text { Papp et al. (12)/ } \\
2015\end{array}$ & $\begin{array}{l}\text { Plaque psoriasis-tofacitinib } 10 \\
\text { or } 5 \mathrm{mg} \text { or placebo, twice daily. }\end{array}$ & $\begin{array}{l}\text { Tofacitinib } \\
5 \text { mg-745; } 10 \\
\text { mg-741; } \\
\text { placebo- } 373\end{array}$ & $\begin{array}{l}\text { At week } 16 \text {, a greater proportion } \\
\text { of patients achieved PGA } \\
\text { responses with tofacitinib } 5 \text { and } \\
10 \text { mg twice daily vs. placebo. }\end{array}$ & $\begin{array}{l}\text { Similar across } \\
\text { groups. Twelve } \\
\text { patients reported } \\
\text { herpes zoster } \\
\text { across the } \\
\text { tofacitinib } \\
\text { treatment groups. }\end{array}$ & $\begin{array}{l}\text { Oral tofacitinib demonstrated } \\
\text { significantly high efficacy as } \\
\text { compared to placebo, during } \\
16 \text { weeks of treatment. }\end{array}$ & Phase 3 \\
\hline & $\begin{array}{l}\text { Mease et al. (15)/ } \\
2017\end{array}$ & $\begin{array}{l}\text { Psoriatic arthritis-tofacitinib } \\
5 \text {-mg twice daily, } 10-\mathrm{mg} \text { twice } \\
\text { daily, adalimumab } 40-\mathrm{mg} \text { once } \\
\text { every } 2 \text { weeks, placebo with a } \\
\text { blinded switch to } 5 \text {-mg } \\
\text { tofacitinib at } 3 \text { months, or } \\
\text { placebo with a blinded switch to } \\
\text { 10-mg tofacitinib at } 3 \text { months. }\end{array}$ & $\begin{array}{l}\text { Tofacitinib } \\
5 \text { mg-107; } 10 \\
\text { mg-104; } \\
\text { adalimumab- 106; } \\
\text { placebo- } 52 \text { (5 mg } \\
\text { switch), } 53 \text { (10 } \\
\text { mg switch). }\end{array}$ & $\begin{array}{l}\text { ACR20 response rates at } \\
\text { month } 3 \text { were } 50 \% \text { in } 5 \text {-mg } \\
\text { tofacitinib group and } 61 \% \text { in } \\
10 \text {-mg tofacitinib group, } 33 \% \text { in } \\
\text { placebo group, } 52 \% \text { in the } \\
\text { adalimumab group. }\end{array}$ & $\begin{array}{l}\text { The rate of } \\
\text { adverse events } \\
\text { was } 66 \% \text { in } 5-m g \\
\text { tofacitinib group, } \\
71 \% \text { in } 10-m g \\
\text { tofacitinib group, } \\
72 \% \text { in } \\
\text { adalimumab } \\
\text { group. }\end{array}$ & $\begin{array}{l}\text { Efficacy of tofacitinib was } \\
\text { superior to placebo at month } \\
3 \text { in patients who previously } \\
\text { had an inadequate response } \\
\text { to conventional synthetic } \\
\text { DMARDs. }\end{array}$ & $\begin{array}{l}\text { 12-month, } \\
\text { double-blind, } \\
\text { active-controlled } \\
\text { and placebo- } \\
\text { controlled, phase } \\
3 \text { trial }\end{array}$ \\
\hline
\end{tabular}




\begin{tabular}{|c|c|c|c|c|c|c|c|}
\hline Drug & Study/year & Setting/Dose & $\begin{array}{l}\text { Number of } \\
\text { patients }\end{array}$ & Response & Adverse effects & Conclusion & Phase \\
\hline & $\begin{array}{l}\text { Gladman et al. } \\
(16) / 2017\end{array}$ & $\begin{array}{l}\text { Psoriatic arthritis-tofacitinib } \\
5 \mathrm{mg} \text { twice daily; } 10 \mathrm{mg} \text { twice } \\
\text { daily; placebo, with a switch to } \\
5 \mathrm{mg} \text { tofacitinib twice daily at } 3 \\
\text { months; or placebo, with a } \\
\text { switch to } 10 \mathrm{mg} \text { tofacitinib twice } \\
\text { daily at } 3 \text { months. }\end{array}$ & $\begin{array}{l}\text { Tofacitinib } \\
5 \text { mg-132; } 10 \\
\text { mg-132; } \\
\text { placebo- } 66 \text { (5 mg } \\
\text { switch), } 65 \text { (10 } \\
\text { mg switch). }\end{array}$ & $\begin{array}{l}\text { ACR20 response- } 50 \% \text { with } \\
5 \text {-mg tofacitinib and } 47 \% \text { with } \\
10-\mathrm{mg} \text { dose, as compared to } \\
24 \% \text { with placebo. }\end{array}$ & $\begin{array}{l}4 \text { serious } \\
\text { infections, } 3 \\
\text { herpes zoster } \\
\text { infections, } 1 \\
\text { myocardial } \\
\text { infarction, and } 1 \\
\text { ischemic stroke. }\end{array}$ & $\begin{array}{l}\text { Tofacitinib was more effective } \\
\text { than placebo over } 3 \text { months } \\
\text { in reducing disease activity. }\end{array}$ & $\begin{array}{l}\text { 6-month } \\
\text { randomized, } \\
\text { placebo- } \\
\text { controlled, } \\
\text { double-blind, } \\
\text { phase } 3 \text { trial }\end{array}$ \\
\hline Baricitinib & $\begin{array}{l}\text { Papp et al. (19)/ } \\
2016\end{array}$ & $\begin{array}{l}\text { Moderate-to-severe psoriasis- } \\
\text { placebo or oral baricitinib at } 2 \text {, } \\
4,8 \text { or } 10 \mathrm{mg} \text { once daily for } 12 \\
\text { weeks. }\end{array}$ & $\begin{array}{l}\text { baricitinib } 2 \text { mg- } \\
32,4 \mathrm{mg}-72,8 \\
\text { mg- } 64,10 \mathrm{mg}- \\
69, \text { Placebo- } 34\end{array}$ & $\begin{array}{l}\text { At week } 12, \text { patients in } 8-\mathrm{mg} \\
(43 \%) \text { and } 10-\mathrm{mg}(54 \%) \\
\text { baricitinib groups achieved } \\
\text { PASI-75 than in placebo group } \\
(17 \%) \text {. } \\
\text { Statistically significant PASI-90 } \\
\text { responses were achieved in } \\
8 \text {-mg and } 10-\mathrm{mg} \text { groups at } 8 \\
\text { and } 12 \text { weeks. }\end{array}$ & $\begin{array}{l}\text { treatment- } \\
\text { emergent } A E \text { rates } \\
\text { were } 44,50,47, \\
58 \text { and } 64 \% \text { for } \\
\text { placebo and } 2-\text {, } \\
4-, 8-\text { and } 10-m g \\
\text { baricitinib groups. }\end{array}$ & $\begin{array}{l}\text { Treatment with baricitinib for } \\
12 \text { weeks achieved significant } \\
\text { improvements in PASI-75. }\end{array}$ & $\begin{array}{l}\text { Phase } 2 \mathrm{~b} \text {, } \\
\text { randomized, } \\
\text { double-blind, } \\
\text { placebo- } \\
\text { controlled, } \\
\text { dose-ranging } \\
\text { study. }\end{array}$ \\
\hline \multirow[t]{3}{*}{$\begin{array}{l}\text { IL-12/23 } \\
\text { inhibitors } \\
\text { Ustekinumab }\end{array}$} & $\begin{array}{l}\text { Phoenix-I (89)/ } \\
2008\end{array}$ & $\begin{array}{l}\text { Moderate-to-severe psoriasis- } \\
\text { Ustekinumab } 45 \mathrm{mg} \text { or } 90 \mathrm{mg} \text { at } \\
\text { weeks } 0,4 \text { and then every } 12 \\
\text { weeks; or placebo at weeks } 0 \\
\text { and } 4 \text {, with subsequent } \\
\text { crossover to ustekinumab at } \\
\text { week } 12 \text {. }\end{array}$ & $\begin{array}{l}\text { Placebo-255; } 45 \\
\text { mg- 255; } 90 \mathrm{mg}- \\
256\end{array}$ & $\begin{array}{l}67.1 \% \text { patients receiving } \\
\text { ustekinumab } 45 \mathrm{mg}, 66.4 \% \\
\text { receiving ustekinumab } 90 \mathrm{mg} \text {, } \\
\text { and } 3.1 \% \text { receiving placebo } \\
\text { achieved PASI } 75 \text { at week } 12 .\end{array}$ & $\begin{array}{l}\text { Adverse events } \\
\text { occurred in } 54.5 \% \\
\text { in ustekinumab } \\
\text { and } 48 \cdot 2 \% \text { in } \\
\text { placebo group. }\end{array}$ & $\begin{array}{l}\text { Ustekinumab seems to be } \\
\text { efficacious for the treatment of } \\
\text { moderate-to-severe psoriasis; } \\
\text { dosing every } 12 \text { weeks } \\
\text { maintains efficacy for at least } \\
\text { a year in most patients. }\end{array}$ & $\begin{array}{l}\text { Phase 3, parallel, } \\
\text { double-blind, } \\
\text { placebo-controlled } \\
\text { study. }\end{array}$ \\
\hline & $\begin{array}{l}\text { Phoenix-II (90)/ } \\
2008\end{array}$ & $\begin{array}{l}\text { Moderate-to-severe psoriasis- } \\
\text { Ustekinumab } 45 \mathrm{mg} \text { or } 90 \mathrm{mg} \text { at } \\
\text { weeks } 0,4 \text { and then every } 12 \\
\text { weeks; or placebo. } \\
\text { Partial responders (patients } \\
\text { achieving } \geq 50 \% \text { but }<75 \% \\
\text { improvement from baseline in } \\
\text { PASI) were re-randomized at } \\
\text { week } 28 \text { to continue dosing } \\
\text { every } 12 \text { weeks or escalate to } \\
\text { dosing every } 8 \text { weeks. }\end{array}$ & $\begin{array}{l}\text { Placebo- } 410 ; 45 \\
\text { mg- 409; } 90 \\
\text { mg-411 }\end{array}$ & $\begin{array}{l}66.7 \% \text { patients receiving } \\
\text { ustekinumab } 45 \mathrm{mg}, 75.7 \% \\
\text { receiving ustekinumab } 90 \mathrm{mg} \text {, } \\
\text { and } 3.7 \% \text { receiving placebo } \\
\text { achieved } 75 \% \text { improvement in } \\
\text { PASI at week } 12 \text {. } \\
\text { More partial responders who } \\
\text { received ustekinumab } 90 \mathrm{mg} \\
\text { every } 8 \text { weeks achieved PASI } \\
75 \text { at week } 52 \text { than those who } \\
\text { received the same dose every } \\
12 \text { weeks. }\end{array}$ & $\begin{array}{l}\text { Serious adverse } \\
\text { events were seen } \\
\text { in } 2 \% \text { patients in } \\
45 \mathrm{mg} \text { group, } \\
1.2 \% \text { in } 90 \mathrm{mg} \\
\text { group, and } 2 \% \text { in } \\
\text { placebo group. }\end{array}$ & $\begin{array}{l}\text { Ustekinumab every } 12 \text { weeks } \\
\text { is effective for most patients } \\
\text { with moderate-to-severe } \\
\text { psoriasis. Intensification of } \\
\text { dosing to once every } 8 \text { weeks } \\
\text { with ustekinumab } 90 \mathrm{mg} \\
\text { might be necessary to elicit a } \\
\text { full response in patients who } \\
\text { only partially respond to the } \\
\text { initial regimen. }\end{array}$ & $\begin{array}{l}\text { Multicentre, phase } \\
3 \text {, double-blind, } \\
\text { placebo-controlled } \\
\text { study. }\end{array}$ \\
\hline & $\begin{array}{l}\text { Griffiths et al. (91)/ } \\
2010\end{array}$ & $\begin{array}{l}\text { Moderate-to-severe psoriasis- } \\
45 \text { or } 90 \text { mg of ustekinumab (at } \\
\text { weeks } 0 \text { and } 4 \text { ) or high-dose } \\
\text { etanercept ( } 50 \text { mg twice weekly } \\
\text { for } 12 \text { weeks) }\end{array}$ & $\begin{array}{l}45 \mathrm{mg}-209 ; 90 \\
\mathrm{mg}-347 ; \\
\text { etanercept-347 }\end{array}$ & $\begin{array}{l}75 \% \text { improvement in the PASI } \\
\text { at week } 12 \text { in } 67.5 \% \text { of patients } \\
\text { receiving } 45 \mathrm{mg} \text { of ustekinumab } \\
\text { and } 73.8 \% \text { of patients receiving } \\
90 \mathrm{mg} \text {, as compared with } \\
56.8 \% \text { of those with etanercept. }\end{array}$ & $\begin{array}{l}\text { One or more } \\
\text { adverse events } \\
\text { occurred in } 66 \% \text { of } \\
\text { patients in } 45 \mathrm{mg} \\
\text { ustekinumab and } \\
69.2 \% \text { in } 90 \mathrm{mg} \\
\text { ustekinumab and } \\
\text { in } 70 \% \text { in } \\
\text { etanercept group. }\end{array}$ & $\begin{array}{l}\text { Efficacy of ustekinumab } 45 \text { or } \\
90 \mathrm{mg} \text { was superior to } \\
\text { high-dose etanercept over a } \\
\text { 12-week period. }\end{array}$ & $\begin{array}{l}\text { Randomized, } \\
\text { multicentre study. }\end{array}$ \\
\hline
\end{tabular}


TABLE 1 | Continued

\begin{tabular}{|c|c|c|c|c|c|c|c|}
\hline Drug & Study/year & Setting/Dose & $\begin{array}{l}\text { Number of } \\
\text { patients }\end{array}$ & Response & Adverse effects & Conclusion & Phase \\
\hline & PSUMMIT I (35) & $\begin{array}{l}\text { Active psoriatic arthritis-45 mg } \\
\text { ustekinumab, } 90 \mathrm{mg} \\
\text { ustekinumab, or placebo at } \\
\text { week } 0 \text {, week } 4 \text {, and every } 12 \\
\text { weeks thereafter. }\end{array}$ & $\begin{array}{l}\text { Placebo- 206; } 45 \\
\text { mg- 205; } 90 \\
\text { mg-204 }\end{array}$ & $\begin{array}{l}\text { More ustekinumab-treated } \\
\text { [42.4\%] in the } 45 \mathrm{mg} \text { group and } \\
{[49.5 \%] \text { in the } 90 \mathrm{mg} \text { group than }} \\
\text { placebo-treated [22.8\%] } \\
\text { patients achieved ACR20 at } \\
\text { week } 24 .\end{array}$ & $\begin{array}{l}\text { Adverse events } \\
\text { were similar in the } \\
\text { ustekinumab } \\
{[41 \cdot 8 \%] \text { and }} \\
\text { placebo groups } \\
{[42 \cdot 0 \%] \text {. }}\end{array}$ & $\begin{array}{l}\text { Ustekinumab significantly } \\
\text { improved active psoriatic } \\
\text { arthritis. }\end{array}$ & $\begin{array}{l}\text { Phase 3, } \\
\text { multicentre, } \\
\text { double-blind, } \\
\text { placebo-controlled } \\
\text { trial }\end{array}$ \\
\hline & PSUMMIT II (36) & $\begin{array}{l}\text { Active Psoriatic Arthritis- } \\
\text { ustekinumab } 45 \mathrm{mg} \text { or } 90 \mathrm{mg} \text { at } \\
\text { week } 0 \text {, week } 4, \mathrm{q} 12 \text { weeks or } \\
\text { placebo at week } 0 \text {, week } 4 \text {, } \\
\text { week } 16 \text { and crossover to } \\
\text { ustekinumab } 45 \mathrm{mg} \text { at week } 24 \text {, } \\
\text { week } 28 \text { and week } 40 \text {. }\end{array}$ & $\begin{array}{l}\text { Placebo- 104; } 45 \\
\text { mg- 103; } 90 \text { mg- } \\
105\end{array}$ & $\begin{array}{l}\text { More ustekinumab-treated } \\
(43.8 \% \text { combined) than } \\
\text { placebo-treated }(20.2 \%) \\
\text { patients achieved ACR20 at } \\
\text { week } 24 \text {; all benefits were } \\
\text { sustained through week } 52 .\end{array}$ & $\begin{array}{l}\text { No unexpected } \\
\text { adverse events } \\
\text { were observed. }\end{array}$ & $\begin{array}{l}\text { Ustekinumab ( } 45 / 90 \mathrm{mg} \text { q12 } \\
\text { weeks) yielded significant and } \\
\text { sustained improvements in } \\
\text { Psoriatic arthritis. }\end{array}$ & $\begin{array}{l}\text { phase 3, } \\
\text { multicentre, } \\
\text { placebo-controlled } \\
\text { trial }\end{array}$ \\
\hline \multirow[t]{3}{*}{$\begin{array}{l}\text { IL-23 inhibitor } \\
\text { Guselkumab }\end{array}$} & VOYAGE-I (92) & $\begin{array}{l}\text { Moderate to severe plaque } \\
\text { psoriasis- guselkumab } 100 \mathrm{mg} \\
\text { (weeks } 0 \text { and } 4 \text {, then every } 8 \\
\text { weeks); placebo/guselkumab } \\
\text { (weeks } 0,4 \text {, and } 12 \text { then } \\
\text { guselkumab at weeks } 16 \text { and } \\
20 \text {, then every } 8 \text { weeks); or } \\
\text { adalimumab ( } 80 \text { mg week } 0 \text {, } \\
40 \text { mg week } 1 \text {, then } 40 \mathrm{mg} \\
\text { every } 2 \text { weeks through week } \\
\text { 47). }\end{array}$ & $\begin{array}{l}\text { Placebo- 174; } 100 \\
\text { mg-329; } \\
\text { adalimumab-334 }\end{array}$ & $\begin{array}{l}\text { Guselkumab was superior to } \\
\text { placebo at week } 16 \text { ( } 73.3 \text { vs. } \\
2.9 \% \text { [PASI-90]). Guselkumab } \\
\text { was also superior to } \\
\text { adalimumab for PASI } 90 \text { at } \\
\text { week } 16 \text { (73.3 vs. } 49.7 \%) \text {, week } \\
24 \text { (80.2 vs. } 53.0 \%) \text {, and week } \\
48 \text { (76.3 vs. } 47.9 \%) .\end{array}$ & $\begin{array}{l}\text { The proportions of } \\
\text { patients with } \\
\text { adverse events } \\
\text { were similar in the } \\
\text { guselkumab and } \\
\text { adalimumab } \\
\text { group. }\end{array}$ & $\begin{array}{l}\text { Guselkumab demonstrated } \\
\text { superior efficacy compared } \\
\text { with adalimumab. }\end{array}$ & $\begin{array}{l}\text { phase 3, } \\
\text { randomized, } \\
\text { double-blind, } \\
\text { placebo- and } \\
\text { active comparator- } \\
\text { controlled } \\
\text { trial }\end{array}$ \\
\hline & VOYAGE-II (93) & $\begin{array}{l}\text { Moderate to severe plaque } \\
\text { psoriasis- Similar to VOYAGE I; } \\
\text { at week 28, guselkumab } \\
\text { PASI90 responders were } \\
\text { rerandomized to guselkumab or } \\
\text { placebo with guselkumab after } \\
\text { loss of response. Placebo } \rightarrow \\
\text { guselkumab responders and } \\
\text { adalimumab responders } \\
\text { received placebo, then } \\
\text { guselkumab after loss of } \\
\text { response. }\end{array}$ & $\begin{array}{l}\text { Placebo- 248; } 100 \\
\text { mg-496; } \\
\text { adalimumab-248 }\end{array}$ & $\begin{array}{l}\text { Guselkumab was superior to } \\
\text { adalimumab and placebo at } \\
\text { week } 16 \text {. From weeks } 28 \text { to } 48 \text {, } \\
\text { better persistence of response } \\
\text { was observed in guselkumab } \\
\text { maintenance vs. withdrawal } \\
\text { groups. Of adalimumab } \\
\text { Non-responders who switched } \\
\text { to guselkumab, } 66.1 \% \\
\text { achieved PASI } 90 \text { at week } 48 \text {. }\end{array}$ & $\begin{array}{l}\text { Adverse events } \\
\text { were comparable } \\
\text { among groups. }\end{array}$ & $\begin{array}{l}\text { Guselkumab is highly effective } \\
\text { maintenance therapy, } \\
\text { including in adalimumab } \\
\text { Non-responders. }\end{array}$ & $\begin{array}{l}\text { phase 3, } \\
\text { double-blind, } \\
\text { placebo- and } \\
\text { active } \\
\text { comparator- } \\
\text { controlled }\end{array}$ \\
\hline & DISCOVER I (37) & Active psoriatic arthritis & $\begin{array}{l}\text { Placebo- } 126 ; \\
100 \text { mg every } 4 \\
\text { weeks- } 128 ; \\
100 \text { mg at } 0 \text { and } 4 \\
\text { weeks, then every } \\
8 \text { weeks- } 127\end{array}$ & $\begin{array}{l}\text { Significantly greater proportions } \\
\text { of patients receiving } \\
\text { guselkumab every 4-week } \\
(59.4 \%) \text { and every } 8 \text {-week } \\
\text { (52.0\%) vs. placebo (22.2\%) } \\
\text { achieved ACR20 at week } 24 \text {. }\end{array}$ & $\begin{array}{l}\text { Serious adverse } \\
\text { events occurred in } \\
\text { none of patients in } \\
\text { guselkumab every } \\
4 \text {-week, } 3.1 \% \text { in } \\
\text { guselkumab every } \\
\text { 8-week, and } 4.0 \% \\
\text { in placebo group. }\end{array}$ & $\begin{array}{l}\text { Guselkumab demonstrated a } \\
\text { favorable benefit-risk profile } \\
\text { and is an effective treatment } \\
\text { option in patients with active } \\
\text { psoriatic arthritis. }\end{array}$ & $\begin{array}{l}\text { Phase-3, } \\
\text { double-blind, } \\
\text { placebo-controlled } \\
\text { study }\end{array}$ \\
\hline
\end{tabular}


TABLE 1 | Continued

\begin{tabular}{|c|c|c|c|c|c|c|c|}
\hline Drug & Study/year & Setting/Dose & $\begin{array}{l}\text { Number of } \\
\text { patients }\end{array}$ & Response & Adverse effects & Conclusion & Phase \\
\hline \multirow[t]{2}{*}{ Tildrakizumab } & reSURFACE I (94) & $\begin{array}{l}\text { Moderate-to-severe chronic } \\
\text { plaque psoriasis- Tildrakizumab } \\
\text { at weeks } 0 \text { and } 4 \text { during part } 1 \\
\text { and at week } 16 \text { during part } 2 \\
\text { (weeks } 12 \text { and } 16 \text { for } \\
\text { participants re-randomized from } \\
\text { placebo to tildrakizumab. }\end{array}$ & $\begin{array}{l}\text { Placebo- } 154100 \\
\text { mg- } 309200 \\
\text { mg-308 }\end{array}$ & $\begin{array}{l}\text { At week } 12,62 \% \text { in } 200 \mathrm{mg} \\
\text { group and } 64 \% \text { in } 100 \mathrm{mg} \\
\text { group achieved PASI } 75 \text {, } \\
\text { compared with } 6 \% \text { in placebo } \\
\text { group. }\end{array}$ & Nasopharyngitis. & $\begin{array}{l}\text { Tildrakizumab } 200 \mathrm{mg} \text { and } \\
100 \text { mg were efficacious } \\
\text { compared with placebo. }\end{array}$ & $\begin{array}{l}\text { Parallel group, } \\
\text { double-blind, } \\
\text { randomized } \\
\text { controlled study }\end{array}$ \\
\hline & reSURFACE II (94) & $\begin{array}{l}\text { Moderate-to-severe chronic } \\
\text { plaque psoriasis- Tildrakizumab } \\
\text { at weeks } 0 \text { and } 4 \text { during part } 1 \\
\text { and at week } 16 \text { during part } 2 \\
\text { (weeks } 12 \text { and } 16 \text { for } \\
\text { participants re-randomized from } \\
\text { placebo to tildrakizumab; } \\
\text { etanercept was given twice } \\
\text { weekly in part } 1 \text { and once } \\
\text { weekly during part 2). }\end{array}$ & $\begin{array}{l}\text { Placebo- } 156100 \\
\text { mg- } 307200 \\
\text { mg-314 } \\
\text { Etanercept-313 }\end{array}$ & $\begin{array}{l}\text { At week } 12,66 \% \text { in } 200 \mathrm{mg} \\
\text { group, and } 61 \% \text { in } 100 \mathrm{mg} \\
\text { group achieved PASI } 75 \text {, } \\
\text { compared with } 6 \% \text { in placebo } \\
\text { group and } 48 \% \text { in the } \\
\text { etanercept group. }\end{array}$ & $\begin{array}{l}\text { The incidence of } \\
\text { severe infections, } \\
\text { malignancies, and } \\
\text { major adverse } \\
\text { cardiovascular } \\
\text { events were low } \\
\text { and similar across } \\
\text { treatment groups. }\end{array}$ & $\begin{array}{l}\text { Tildrakizumab } 200 \mathrm{mg} \text { and } \\
100 \mathrm{mg} \text { were efficacious } \\
\text { compared with placebo and } \\
\text { etanercept and were well } \\
\text { tolerated. }\end{array}$ & $\begin{array}{l}\text { Parallel group, } \\
\text { double-blind, } \\
\text { randomized } \\
\text { controlled study }\end{array}$ \\
\hline Risankizumab & $\begin{array}{l}\text { UltIMMa-1 and } \\
\text { UltIMMa-2 (95) }\end{array}$ & $\begin{array}{l}\text { Moderate-to-severe chronic } \\
\text { plaque psoriasis-150 mg } \\
\text { risankizumab, } 45 \mathrm{mg} \text { or } 90 \mathrm{mg} \\
\text { ustekinumab or placebo. } \\
\text { Following } 16 \text {-week double-blind } \\
\text { treatment period (part A), } \\
\text { patients initially assigned to } \\
\text { placebo switched to } 150 \mathrm{mg} \\
\text { risankizumab at week } 16 \text {; other } \\
\text { patients continued their } \\
\text { originally randomized treatment } \\
\text { (part B, double-blind, weeks } \\
\text { 16-52). Study drug was } \\
\text { administered subcutaneously at } \\
\text { weeks } 0 \text { and } 4 \text { during part A } \\
\text { and at weeks } 16,28 \text {, and } 40 \\
\text { during part B. }\end{array}$ & $\begin{array}{l}\text { UltIMMa-1 - } \\
\text { Placebo- 102; } 150 \\
\text { mg-304; } \\
\text { ustekinumab-100 } \\
\text { UltIMMa-2- } \\
\text { Placebo-98; } 150 \\
\text { mg-294; } \\
\text { Ustekinumab- } 99\end{array}$ & $\begin{array}{l}\text { At week } 16 \text { of UltIMMa-1, PASI } \\
90 \text { was achieved by } 75.3 \% \\
\text { patients receiving risankizumab } \\
\text { vs. } 4.9 \% \text { receiving placebo and } \\
42.0 \% \text { receiving ustekinumab. } \\
\text { At week } 16 \text { of UltIMMa-2, PASI } \\
90 \text { was achieved by } 74.8 \% \\
\text { patients receiving risankizumab } \\
\text { vs. } 2.0 \% \text { receiving placebo and } \\
47.5 \% \text {. }\end{array}$ & $\begin{array}{l}\text { The frequency of } \\
\text { treatment- } \\
\text { emergent adverse } \\
\text { events in } \\
\text { UltIMMa-1 and } \\
\text { UltIMMa-2 was } \\
\text { similar across } \\
\text { risankizumab, } \\
\text { placebo, } \\
\text { ustekinumab, and } \\
\text { placebo to } \\
\text { risankizumab } \\
\text { groups. }\end{array}$ & $\begin{array}{l}\text { Risankizumab showed } \\
\text { superior efficacy to both } \\
\text { placebo and ustekinumab. }\end{array}$ & $\begin{array}{l}\text { Phase 3, } \\
\text { randomized, } \\
\text { double-blind, } \\
\text { placebo-controlled } \\
\text { and active } \\
\text { comparator- } \\
\text { controlled } \\
\text { trials }\end{array}$ \\
\hline $\begin{array}{l}\text { IL-36 inhibitor } \\
\text { Spesolimab }\end{array}$ & Bachelez et al. (96) & $\begin{array}{l}\text { Generalized Pustular Psoriasis- } \\
\text { single } 900-\mathrm{mg} \text { intravenous dose } \\
\text { of spesolimab or placebo. } \\
\text { Patients in both groups received } \\
\text { an open-label dose of } \\
\text { spesolimab on day } 8 \text {, an } \\
\text { open-label dose of spesolimab } \\
\text { as a rescue medication after } \\
\text { day } 8 \text {, or both and were } \\
\text { followed to week } 12 \text {. }\end{array}$ & $\begin{array}{l}\text { Spesolimab } 900 \\
\text { mg- 35; placebo- } \\
18\end{array}$ & $\begin{array}{l}\text { At week } 1,54 \% \text { in the } \\
\text { spesolimab group had a } \\
\text { pustulation sub-score of } 0 \text {, as } \\
\text { compared with } 6 \% \text { in the } \\
\text { placebo group. }\end{array}$ & $\begin{array}{l}\text { Drug reactions-2 } \\
\text { patients. } \\
\text { (drug-induced } \\
\text { hepatic injury- 1); } \\
\text { infections-17\% } \\
\text { through the first } \\
\text { week; antidrug } \\
\text { antibodies-46\%. }\end{array}$ & $\begin{array}{l}\text { Spesolimab resulted in a } \\
\text { higher incidence of lesion } \\
\text { clearance at } 1 \text { week than } \\
\text { placebo but was associated } \\
\text { with infections and systemic } \\
\text { drug reactions. }\end{array}$ & $\begin{array}{l}\text { Phase } 2 \\
\text { randomized trial }\end{array}$ \\
\hline
\end{tabular}


to the production of IL-1 $\beta$ (48). Higher caspase-1 and IL$1 \beta$ levels has been observed in patients with psoriasis that normalized after treatment with TNF- $\alpha$ (48). Anti-IL1 agents such as anakinra, canakinumab and gevokizumab have shown efficacy in psoriasis. Anakinra, a recombinant IL-1 receptor antagonist (IL-1Ra) inhibits both IL- $1 \alpha$ and IL- $\beta$ and has shown efficacy in pustular psoriasis and deficiency of IL-1 receptor antagonist (DIRA) variant (49). However, the partial responses observed suggest role of other cytokines of IL-1 family such as IL$36(49,50)$. Canakinumab, an anti-IL- $\beta$ antibody has also shown beneficial effects in GPP (51). Gevokizumab, another novel IL$1 \beta$ antagonist has shown its efficacy in GPP (52). In 2 patients of GPP, 79 and 65\% improvement in GPP scores was observed after 4 weeks (52). Thus, IL-1 inhibitors particularly IL- $1 \beta$ could be potentially efficacious in management of psoriasis especially pustular psoriasis, though larger studies are needed.

\section{ROR $\gamma$ T ANTAGONISTS}

$\mathrm{ROR} \gamma \mathrm{T}$ is an important transcription factor required for the differentiation of Th17 cells and regulates the expression of Th17 cytokines i.e., IL-17A, IL-17F, IL-22 and IL-23 receptor (53). Thus, ROR $\gamma \mathrm{T}$ inhibition seems to be an effective strategy in therapeutics of psoriasis. VTP-43742, an oral ROR $\gamma \mathrm{T}$ inhibitor is undergoing phase III study in treatment of plaque psoriasis. In a phase IIa study, 29 and $23 \%$ reduction in PASI was observed at 4 weeks in patients receiving $700 \mathrm{mg}$ and $350 \mathrm{mg}$ of VTP43742 respectively along with $75 \%$ reduction in IL-17A and IL$17 \mathrm{~F}$ levels in both groups (54). Side effects included headache, flushing, elevated liver enzymes and nausea. Other agents such as JTE-451 and ABBV-157, oral ROR $\gamma \mathrm{T}$ inhibitors are currently in phase 2 and phase 1 of development respectively, for the treatment of moderate to severe psoriasis. New systemic and topical ROR $\gamma \mathrm{T}$ inhibitors may be the potential candidates for the treatment of psoriasis (55).

\section{TYK2 INHIBITORS}

The TYK2, a JAK family gene, has been associated with psoriasis susceptibility genes and loss of function mutation is associated with various cytokine signaling defects that are implicated in psoriasis pathogenesis $(56,57)$. Individuals with these mutations have been found to be unaffected by immunemediated inflammatory diseases without being susceptible to lifethreatening infections (58). These observations suggested that TYK2 inhibitors may be a safe therapeutic target. BMS-986165 is an oral highly selective TYK2 inhibitor and inhibit STAT1 and STAT3 phosphorylation in peripheral blood mononuclear cells stimulated with IFN- $\alpha$ and IL-23 (6). BMS-986165 has shown good efficacy in psoriasis in phase II trials at doses $3 \mathrm{mg}, 6 \mathrm{mg}$ and $12 \mathrm{mg}$ daily (59). Common adverse effects include headache, nausea, diarrhea, and upper respiratory tract infections (59). Phase III trials in plaque psoriasis and phase II trial in psoriatic arthritis are currently undergoing (6). Another selective TYK2 inhibitor, PF-06826647, is also being tested in moderate-to-severe psoriasis in an ongoing phase II clinical trial (NCT03895372)
(6). Brepocitinib (formerly known as PF-06700841) is not a selective TYK2 inhibitor (rather a potent TYK2/JAK1 inhibitor), has shown good efficacy in phase II trials in psoriasis with few minor adverse effects, except thrombocytopenia and decreased reticulocyte count (60). A phase IIb study is currently undergoing to evaluate the efficacy and safety in psoriatic arthritis (6). A topical formulation is also being tested in mild to moderate psoriasis. These small molecules have advantages like oral route of administration, decreased cost, less immunologic adverse events as compared to biologics.

\section{SPHINGTOSINE-1-PHOSPHATE RECEPTOR 1 (S1PR1) ANTAGONIST}

Sphingosine-1-phosphate (S1P) is involved in cell proliferation and survival, migration, inflammation and angiogenesis $(61,62)$. S1P inhibits the keratinocyte proliferations and increase cell differentiation (63). Ponesimod, an oral S1P receptor 1 antagonist leads to the downregulation of $\mathrm{S} 1 \mathrm{P}$ receptor and prevent migration of lymphocytes from lymph nodes to skin in psoriasis (64). In a phase 2 study, PASI75 was achieved in 46 and $48 \%$ of patients receiving ponesimod $20 \mathrm{mg}$ and $40 \mathrm{mg}$ respectively as compared to placebo at 16-weeks and the improvement continued till 28 weeks (65). However, effect is not maintained after drug discontinuation due to its rapid elimination within 1 week. Adverse effects include transaminitis, shortness of breath, dizziness and may cause conduction abnormalities, thus contraindicated in patients with cardiac disease (65).

\section{A3 ADENOSINE RECEPTOR AGONIST}

A3 adenosine receptors are G-protein coupled receptors involved in various intracellular pathways. These receptors have been found to be highly expressed on peripheral mononuclear cells in psoriasis patients (66). Piclidenoson, an oral A3 adenosine receptor agonist has been found to downregulate NF-кB signaling pathway and pro-inflammatory cytokines such as TNF- $\alpha$, IL-6 and IL-12, and inhibit T-lymphocyte proliferation (67). In a phase II trial, a significant reduction in PASI was observed at 12 weeks as compared to placebo and drug was well tolerated (67). Currently, the drug is in phase III trials.

\section{mTOR INHIBITORS}

The PI3-K/Akt/mTORC1 cascade acts as a regulator of epidermal homeostasis (68). Akt has been shown to be highly activated in skin of psoriatic lesions, except in the basal layer and mTOR, expression is found to be increased in lesional and Non-lesional skin of psoriasis patients $(69,70)$. An animal model study showed that the PUVA treatment led to improvement in psoriasis and normalization of mTORC1 signaling (71). This suggested a pathophysiological role of mTORC1 signaling in psoriasis. The increased expression of mTORC1 may have a role in increased proliferation of keratinocytes and decreased differentiation. During normal 
keratinization, mTORC1 signaling pathway is inactivated as the keratinocyte differentiation occurs (72). mTORC1 signaling also plays important roles in the innate and adaptive immunity $(72,73)$. Aberrant mTORC1 signaling was found in peripheral blood mononuclear cells (PBMCs) of psoriasis patients (74). Rapamycin, a mTOR inhibitor, has been used in few patients with psoriasis due to its antiproliferative and immunosuppressive actions (75). Everolimus was also used successfully in a psoriasis patient along with tacrolimus (76). Topical rapamycin has also been used in psoriasis showing clinical improvement (77). Thus, oral and topical mTOR inhibitors may be a successful therapeutic strategy in psoriasis and further research exploring the role of mTOR pathway as therapeutic target is warranted.

\section{FUTURE PERSPECTIVE}

Recent advances in understanding the pathogenesis of the psoriasis has led to the development of newer therapies such as biologics and other small molecules. However, apert from the therapeutic options discussed, various other cells and pathways are implicated in the pathogenesis such as role of natural killer cells, regulatory T-cells and mesenchymal stem cells (MSCs). The regulatory T-cells have been found increased in lesional skin of psoriasis patients. Similarly, IL-10-producing regulatory $\mathrm{B}$ cells of psoriasis patients were reduced in number and showed decreased IL-10 production. MSCs have been implicated in the psoriasis pathogenesis and may serve as potential therapeutic target. MSCs have immunomodulatory properties and affect Th1 and Th17 lymphocytic inhibition in psoriatic skin (78). These MSCs have also been found to have pleiotropic effects of biologic therapy in psoriasis (79). MSCs based therapy has been tried in few patients with psoriasis with successful outcomes (80-82). However, larger studies are still needed to fully explore the role of these cells as a therapeutic option. Another class of drug i.e., selective serotonin reuptake inhibitors (SSRIs) have been found to be beneficial in psoriasis due to their anti-inflammatory properties and reduction in cytokine levels (83). Moreover, these agents

\section{REFERENCES}

1. Michalek IM, Loring B, John SM. A systematic review of worldwide epidemiology of psoriasis. J Eur Acad Dermatol Venereol. (2017) 31:20512. doi: $10.1111 / \mathrm{jdv} .13854$

2. Armstrong AW, Read C. Pathophysiology, clinical presentation, and treatment of psoriasis: a review. Jama. (2020) 323:194560. doi: 10.1001/jama.2020.4006

3. Augustin M, Radtke MA. Quality of life in psoriasis patients. Expert Rev Pharmacoecon Outcomes Res. (2014) 14:55968. doi: 10.1586/14737167.2014.914437

4. Davidovici BB, Sattar N, Prinz J, Puig L, Emery P, Barker JN, et al. Psoriasis and systemic inflammatory diseases: potential mechanistic links between skin disease and co-morbid conditions. J Invest Dermatol. (2010) 130:178596. doi: 10.1038/jid.2010.103

5. Hawkes JE, Chan TC, Krueger JG. Psoriasis pathogenesis and the development of novel targeted immune therapies. J Allergy Clin Immunol. (2017) 140:64553. doi: 10.1016/j.jaci.2017.07.004 prevent T-cell proliferation by reduced antigen presentation by dendritic cells and causes inflammatory cell apoptosis (83). Role of proanthocyanidins having antioxidant, antiproliferative, antiangiogenic and anti-inflammatory properties as an therapeutic option needs to be investigated as oxidative stress plays an important role in the pathogenesis of psoriasis (84). A potent and selective NF-kB inducing kinase (NIK) inhibitor has been found effective in imiquimod induced psoriasis in animal model, highlighting the potential of newer strategy for the treatment of psoriasis (85). Mutations in CARD14 have been found in psoriasis patients (86). Such genetic associations indicate a role in immune regulatory pathways involved in psoriasis. Such observations may help in the better knowledge of psoriasis susceptibility genes and individualized approaches in management of psoriasis. In addition, the role of keratinocytes as initiators of psoriatic inflammation might further shift the focus to topical treatments. Further studies are needed to obtain better insights in the immunopathogenesis of the disease that may lead to the development of more targeted and more effective therapies.

\section{CONCLUSION}

Many novel systemic and topical therapies are currently in development. The success of these agents depends on the efficacy and safety of these drugs in future studies. Better understanding of inflammatory pathways involved the pathogenesis and newer discoveries may lead to the effective therapeutic strategies in management of psoriasis.

\section{AUTHOR CONTRIBUTIONS}

VT: study design, acquisition, analysis or interpretation of data, and drafting of the manuscript. RM: study concept and design, acquisition, analysis or interpretation of data, drafting of the manuscript, critical revision of the manuscript for important intellectual content, statistical analysis, administrative, technical, or material support, and study supervision. All authors contributed to the article and approved the submitted version.
6. Nogueira M, Puig L, Torres T. JAK inhibitors for treatment of psoriasis: focus on selective TYK2 inhibitors. Drugs. (2020) 80:341-52. doi: 10.1007/s40265-020-01261-8

7. Di Cesare A, Di Meglio P, Nestle FO. The IL-23/Th17 axis in the immunopathogenesis of psoriasis. J Invest Dermatol. (2009) 129:133950. doi: 10.1038/jid.2009.59

8. Harrison DA. The Jak/STAT pathway. Cold Spring Harb Perspect Biol. (2012) 4:a011205. doi: 10.1101/cshperspect.a011205

9. Hald A, Andrés RM, Salskov-Iversen ML, Kjellerup RB, Iversen L, Johansen C. STAT1 expression and activation is increased in lesional psoriatic skin. $B r \quad J$ Dermatol. (2013) 168:302-10. doi: 10.1111/bjd. 12049

10. Calautti E, Avalle L, Poli V. Psoriasis: a STAT3-Centric view. Int J Mol Sci. (2018) 19:171. doi: 10.3390/ijms19010171

11. Sano S, Chan KS, Carbajal S, Clifford J, Peavey M, Kiguchi K, et al. Stat3 links activated keratinocytes and immunocytes required for development of psoriasis in a novel transgenic mouse model. Nat Med. (2005) 11:439. doi: $10.1038 / \mathrm{nm} 1162$ 
12. Papp KA, Menter MA, Abe M, Elewski B, Feldman SR, Gottlieb AB, et al. Tofacitinib, an oral Janus kinase inhibitor, for the treatment of chronic plaque psoriasis: results from two randomized, placebo-controlled, phase III trials. $\mathrm{Br}$ J Dermatol. (2015) 173:949-61. doi: 10.1111/bjd.14018

13. Bissonnette R, Iversen L, Sofen H, Griffiths CE, Foley P, Romiti R, et al. Tofacitinib withdrawal and retreatment in moderate-to-severe chronic plaque psoriasis: a randomized controlled trial. Br J Dermatol. (2015) 172:1395406. doi: 10.1111/bjd.13551

14. Papp KA, Krueger JG, Feldman SR, Langley RG, Thaci D, Torii H, et al. Tofacitinib, an oral Janus kinase inhibitor, for the treatment of chronic plaque psoriasis: long-term efficacy and safety results from 2 randomized phase-III studies and 1 open-label long-term extension study. J Am Acad Dermatol. (2016) 74:841-50. doi: 10.1016/j.jaad.2016.01.013

15. Mease P, Hall S, FitzGerald O, van der Heijde D, Merola JF, Avila-Zapata F, et al. Tofacitinib or adalimumab vs. placebo for psoriatic arthritis. $N$ Engl J Med. (2017) 377:1537-50. doi: 10.1056/NEJMoa1615975

16. Gladman D, Rigby W, Azevedo VF, Behrens F, Blanco R, Kaszuba A, et al. Tofacitinib for psoriatic arthritis in patients with an inadequate response to TNF inhibitors. $N$ Engl J Med. (2017) 377:1525-36. doi: 10.1056/NEJMoa1615977

17. Hosking AM, Juhasz M, Mesinkovska NA. Topical Janus kinase inhibitors: a review of applications in dermatology. J Am Acad Dermatol. (2018) 79:53544. doi: 10.1016/j.jaad.2018.04.018

18. Berekmeri A, Mahmood F, Wittmann M, Helliwell P. Tofacitinib for the treatment of psoriasis and psoriatic arthritis. Expert Rev Clin Immunol. (2018) 14:719-30. doi: 10.1080/1744666X.2018.1512404

19. Papp KA, Menter MA, Raman M, Disch D, Schlichting DE, Gaich C, et al. A randomized phase $2 \mathrm{~b}$ trial of baricitinib, an oral Janus kinase (JAK) 1/JAK2 inhibitor, in patients with moderate-to-severe psoriasis. Br J Dermatol. (2016) 174:1266-76. doi: 10.1111/bjd.14403

20. Bissonnette R, Luchi M, Fidelus-Gort R, Jackson S, Zhang H, Flores R, et al. A randomized, double-blind, placebo-controlled, dose-escalation study of the safety and efficacy of INCB039110, an oral Janus kinase 1 inhibitor, in patients with stable, chronic plaque psoriasis. J Dermatolog Treat. (2016) 27:332-8. doi: 10.3109/09546634.2015.1115819

21. Schmieder GJ, Draelos ZD, Pariser DM, Banfield C, Cox L, Hodge M, et al. Efficacy and safety of the Janus kinase 1 inhibitor PF-04965842 in patients with moderate-to-severe psoriasis: phase II, randomized, double-blind, placebocontrolled study. Br J Dermatol. (2018) 179:54-62. doi: 10.1111/bjd.16004

22. Ludbrook VJ, Hicks KJ, Hanrott KE, Patel JS, Binks MH, Wyres MR, et al. Investigation of selective JAK1 inhibitor GSK2586184 for the treatment of psoriasis in a randomized placebo-controlled phase IIa study. Br J Dermatol. (2016) 174:985-95. doi: 10.1111/bjd.14399

23. Mease P, Coates LC, Helliwell PS, Stanislavchuk M, Rychlewska-Hanczewska A, Dudek A, et al. Efficacy and safety of filgotinib, a selective Janus kinase 1 inhibitor, in patients with active psoriatic arthritis (EQUATOR): results from a randomised, placebo-controlled, phase 2 trial. Lancet. (2018) 392:236777. doi: 10.1016/S0140-6736(18)32483-8

24. Papp K, Pariser D, Catlin M, Wierz G, Ball G, Akinlade B, et al. A phase 2a randomized, double-blind, placebo-controlled, sequential dose-escalation study to evaluate the efficacy and safety of ASP015K, a novel Janus kinase inhibitor, in patients with moderate-to-severe psoriasis. Br J Dermatol. (2015) 173:767-76. doi: 10.1111/bjd.13745

25. Oppmann B, Lesley R, Blom B, Timans JC, Xu Y, Hunte B, et al. Novel p19 protein engages IL-12p40 to form a cytokine, IL-23, with biological activities similar as well as distinct from IL-12. Immunity. (2000) 13:71525. doi: 10.1016/S1074-7613(00)00070-4

26. Lee E, Trepicchio WL, Oestreicher JL, Pittman D, Wang F, Chamian $\mathrm{F}$, et al. Increased expression of interleukin $23 \mathrm{p} 19$ and p40 in lesional skin of patients with psoriasis vulgaris. J Exp Med. (2004) 199:12530. doi: 10.1084/jem.20030451

27. Yawalkar N, Tscharner GG, Hunger RE, Hassan AS. Increased expression of IL-12p70 and IL-23 by multiple dendritic cell and macrophage subsets in plaque psoriasis. J Dermatol Sci. (2009) 54:99-105. doi: 10.1016/j.jdermsci.2009.01.003

28. Pastor-Fernández G, Mariblanca IR, Navarro MN. Decoding IL-23 signaling cascade for new therapeutic opportunities. Cells. (2020) 9:2044. doi: 10.3390/cells9092044
29. Yang K, Oak ASW, Elewski BE. Use of IL-23 inhibitors for the treatment of plaque psoriasis and psoriatic arthritis: a comprehensive review. Am J Clin Dermatol. (2021) 22:173-92. doi: 10.1007/s40257-020-00578-0

30. Sawyer LM, Malottki K, Sabry-Grant C, Yasmeen N, Wright E, Sohrt A, et al. Assessing the relative efficacy of interleukin-17 and interleukin-23 targeted treatments for moderate-to-severe plaque psoriasis: a systematic review and network meta-analysis of PASI response. PLoS One. (2019) 14:e0220868. doi: 10.1371/journal.pone.0220868

31. Kimball AB, Gordon KB, Fakharzadeh S, Yeilding N, Szapary PO, Schenkel $\mathrm{B}$, et al. Long-term efficacy of ustekinumab in patients with moderate-tosevere psoriasis: results from the PHOENIX 1 trial through up to 3 years. $\mathrm{Br} \mathrm{J}$ Dermatol. (2012) 166:861-72. doi: 10.1111/j.1365-2133.2012.10901.x

32. Maliyar K, O’Toole A, Gooderham MJ. Long-term single center experience in treating plaque psoriasis with guselkumab. J Cutan Med Surg. (2020) 24:588-95. doi: 10.1177/1203475420932514

33. Warren RB, Carrascosa JM, Fumero E, Schoenenberger A, Lebwohl MG, Szepietowski JC, et al. Time to relapse after tildrakizumab withdrawal in patients with moderate-to-severe psoriasis who were responders at week 28: post hoc analysis through 64 weeks from reSURFACE 1 trial. J Eur Acad Dermatol Venereol. (2021) 35:919-27. doi: 10.1111/jdv.16964

34. Blauvelt A, Leonardi CL, Gooderham M, Papp KA, Philipp S, Wu JJ, et al. Efficacy and safety of continuous risankizumab therapy vs. treatment withdrawal in patients with moderate to severe plaque psoriasis: a phase 3 randomized clinical trial. JAMA Dermatol. (2020) 156:64958. doi: 10.1001/jamadermatol.2020.0723

35. McInnes IB, Kavanaugh A, Gottlieb AB, Puig L, Rahman P, Ritchlin $\mathrm{C}$, et al. Efficacy and safety of ustekinumab in patients with active psoriatic arthritis: 1 year results of the phase 3, multicentre, doubleblind, placebo-controlled PSUMMIT 1 trial. Lancet. (2013) 382:7809. doi: 10.1016/S0140-6736(13)60594-2

36. Ritchlin C, Rahman P, Kavanaugh A, McInnes IB, Puig L, Li S, et al. Efficacy and safety of the anti-IL-12/23 p40 monoclonal antibody, ustekinumab, in patients with active psoriatic arthritis despite conventional nonbiological and biological anti-tumour necrosis factor therapy: 6-month and 1-year results of the phase 3, multicentre, double-blind, placebocontrolled, randomised PSUMMIT 2 trial. Ann Rheum Dis. (2014) 73:9909. doi: 10.1136/annrheumdis-2013-204655

37. Deodhar A, Helliwell PS, Boehncke WH, Kollmeier AP, Hsia EC, Subramanian $\mathrm{RA}$, et al. Guselkumab in patients with active psoriatic arthritis who were biologic-naive or had previously received $\mathrm{TNF} \alpha$ inhibitor treatment (DISCOVER-1): a double-blind, randomised, placebo-controlled phase 3 trial. Lancet. (2020) 395:1115-25. doi: 10.1016/S0140-6736(20)30265-8

38. Madonna S, Girolomoni G, Dinarello CA, Albanesi C. The Significance of IL-36 hyperactivation and IL-36R targeting in psoriasis. Int J Mol Sci. (2019) 20:3318. doi: 10.3390/ijms20133318

39. D’Erme AM, Wilsmann-Theis D, Wagenpfeil J, Hölzel M, Ferring-Schmitt S,

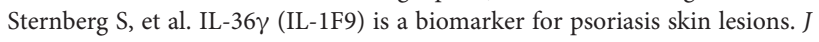
Invest Dermatol. (2015) 135:1025-32. doi: 10.1038/jid.2014.532

40. Johnston A, Xing X, Wolterink L, Barnes DH, Yin Z, Reingold L, et al. IL-1 and IL-36 are dominant cytokines in generalized pustular psoriasis. J Allergy Clin Immunol. (2017) 140:109-20. doi: 10.1016/j.jaci.2016. 08.056

41. Hussain S, Berki DM, Choon SE, Burden AD, Allen MH, Arostegui JI, et al. IL36RN mutations define a severe autoinflammatory phenotype of generalized pustular psoriasis. J Allergy Clin Immunol. (2015) 135:106770.e9. doi: 10.1016/j.jaci.2014.09.043

42. Su Z, Paulsboe S, Wetter J, Salte K, Kannan A, Mathew S, et al. IL36 receptor antagonistic antibodies inhibit inflammatory responses in preclinical models of psoriasiform dermatitis. Exp Dermatol. (2019) 28:11320. doi: 10.1111/exd.13841

43. Mahil SK, Catapano M, Di Meglio P, Dand N, Ahlfors H, Carr IM, et al. An analysis of IL-36 signature genes and individuals with IL1RL2 knockout mutations validates IL-36 as a psoriasis therapeutic target. Sci Transl Med. (2017) 9:eaan2514. doi: 10.1126/scitranslmed.aan2514

44. Todorović V, Su Z, Putman CB, Kakavas SJ, Salte KM, McDonald HA, et al. Small molecule IL-36 $\gamma$ Antagonist as a novel therapeutic approach for plaque psoriasis. Sci Rep. (2019) 9:9089. doi: 10.1038/s41598-019-45 626-w 
45. Ratnarajah K, Jfri A, Litvinov IV, Netchiporouk E. Spesolimab: a novel treatment for pustular psoriasis. J Cutan Med Surg. (2020) 24:199200. doi: $10.1177 / 1203475419888862$

46. Iznardo H, Puig L. The interleukin-1 family cytokines in psoriasis: pathogenetic role and therapeutic perspectives. Expert Rev Clin Immunol. (2021) 17:187-99. doi: 10.1080/1744666X.2021.1886081

47. Cai Y, Xue F, Quan C, Qu M, Liu N, Zhang Y, et al. A critical role of the IL-1 $\beta$ IL-1R Signaling Pathway in Skin Inflammation and Psoriasis Pathogenesis. J Invest Dermatol. (2019) 139:146-56. doi: 10.1016/j.jid.2018.07.025

48. Verma D, Fekri SZ, Sigurdardottir G, Bivik Eding C, Sandin C, Enerbäck C. Enhanced inflammasome activity in patients with psoriasis promotes systemic inflammation. J Invest Dermatol. (2021) 141:58695.e5. doi: 10.1016/j.jid.2020.07.012

49. Tauber M, Viguier M, Alimova E, Petit A, Lioté F, Smahi A, et al. Partial clinical response to anakinra in severe palmoplantar pustular psoriasis. $\mathrm{Br}$ J Dermatol. (2014) 171:646-9. doi: 10.1111/bjd.13012

50. Wang WM, Jin HZ. Biologics in the treatment of pustular psoriasis. Expert Opin Drug Saf. (2020) 19:969-80. doi: 10.1080/14740338.2020.1785427

51. Skendros P, Papagoras C, Lefaki I, Giatromanolaki A, Kotsianidis I, Speletas $\mathrm{M}$, et al. Successful response in a case of severe pustular psoriasis after interleukin-1 $\beta$ inhibition. Br J Dermatol. (2017) 176:2125. doi: 10.1111/bjd.14685

52. Mansouri B, Richards L, Menter A. Treatment of two patients with generalized pustular psoriasis with the interleukin-1 $\beta$ inhibitor gevokizumab. $\mathrm{Br} J$ Dermatol. (2015) 173:239-41. doi: 10.1111/bjd.13614

53. Tang L, Yang X, Liang Y, Xie H, Dai Z, Zheng G. Transcription factor retinoidrelated orphan receptor $\gamma \mathrm{t}$ : a promising target for the treatment of psoriasis. Front Immunol. (2018) 9:1210. doi: 10.3389/fimmu.2018.01210

54. Pandya VB, Kumar S, Sachchidanand, Sharma R, Desai RC. Combating autoimmune diseases with retinoic acid receptor-related orphan receptor- $\gamma$ (ROR $\gamma$ or RORc) Inhibitors: Hits and Misses. J Med Chem. (2018) 61:1097695. doi: 10.1021/acs.jmedchem.8b00588

55. Imura C, Ueyama A, Sasaki Y, Shimizu M, Furue Y, Tai N, et al. A novel $\operatorname{ROR} \gamma \mathrm{t}$ inhibitor is a potential therapeutic agent for the topical treatment of psoriasis with low risk of thymic aberrations. J Dermatol Sci. (2019) 93:176-85. doi: 10.1016/j.jdermsci.2019.03.002

56. Strange A, Capon F, Spencer CC, Knight J, Weale ME, Allen MH, et al. A genome-wide association study identifies new psoriasis susceptibility loci and an interaction between HLA-C and ERAP1. Nat Genet. (2010) 42:98590. doi: 10.1038/ng.694

57. Minegishi Y, Saito M, Morio T, Watanabe K, Agematsu K, Tsuchiya S, et al. Human tyrosine kinase 2 deficiency reveals its requisite roles in multiple cytokine signals involved in innate and acquired immunity. Immunity. (2006) 25:745-55. doi: 10.1016/j.immuni.2006.09.009

58. Dendrou CA, Cortes A, Shipman L, Evans HG, Attfield KE, Jostins L, et al. Resolving TYK2 locus genotype-to-phenotype differences in autoimmunity. Sci Transl Med. (2016) 8:363ra149. doi: 10.1126/scitranslmed.aag1974

59. Papp K, Gordon K, Thaçi D, Morita A, Gooderham M, Foley P, et al. Phase 2 trial of selective tyrosine kinase 2 inhibition in psoriasis. N Engl J Med. (2018) 379:1313-21. doi: 10.1056/NEJMoa1806382

60. Forman S, Pariser D, Poulin Y, Vincent M, Gilbert S, Kieras E, et al. editors. A Phase 2A, randomised, double-blind, placebo-controlled study to evaluate efficacy and safety of PF-06700841 in patients with moderate-to-severe plaque psoriasis. Experimental Dermatology. NJ, USA: Wiley (2018) 27:37.

61. Kunkel GT, Maceyka M, Milstien S, Spiegel S. Targeting the sphingosine-1phosphate axis in cancer, inflammation and beyond. Nat Rev Drug Discov. (2013) 12:688-702. doi: 10.1038/nrd4099

62. Cyster JG, Schwab SR. Sphingosine-1-phosphate and lymphocyte egress from lymphoid organs. Annu Rev Immunol. (2012) 30:69-94. doi: 10.1146/annurev-immunol-020711-075011

63. Schüppel M, Kürschner U, Kleuser U, Schäfer-Korting M, Kleuser B. Sphingosine 1-phosphate restrains insulin-mediated keratinocyte proliferation via inhibition of Akt through the S1P2 receptor subtype. $J$ Invest Dermatol. (2008) 128:1747-56. doi: 10.1038/sj.jid.5701259

64. Piali L, Froidevaux S, Hess P, Nayler O, Bolli MH, Schlosser E, et al. The selective sphingosine 1-phosphate receptor 1 agonist ponesimod protects against lymphocyte-mediated tissue inflammation. J Pharmacol Exp Ther. (2011) 337:547-56. doi: 10.1124/jpet.110.176487
65. Vaclavkova A, Chimenti S, Arenberger P, Holló P, Sator PG, Burcklen M, et al. Oral ponesimod in patients with chronic plaque psoriasis: a randomised, double-blind, placebo-controlled phase 2 trial. Lancet. (2014) 384:203645. doi: 10.1016/S0140-6736(14)60803-5

66. Jacobson KA, Merighi S, Varani K, Borea PA, Baraldi S, Aghazadeh Tabrizi M, et al. A(3) adenosine receptors as modulators of inflammation: from medicinal chemistry to therapy. Med Res Rev. (2018) 38:103172. doi: $10.1002 /$ med. 21456

67. Cohen S, Barer F, Itzhak I, Silverman MH, Fishman P. Inhibition of IL-17 and IL-23 in human keratinocytes by the $\mathrm{A}(3)$ adenosine receptor agonist piclidenoson. J Immunol Res. (2018) 2018:2310970. doi: 10.1155/2018/2310970

68. Manning BD, Cantley LC. AKT/PKB signaling: navigating downstream. Cell. (2007) 129:1261-74. doi: 10.1016/j.cell.2007.06.009

69. Madonna S, Scarponi C, Pallotta S, Cavani A, Albanesi C. Anti-apoptotic effects of suppressor of cytokine signaling 3 and 1 in psoriasis. Cell Death Dis. (2012) 3:e334. doi: 10.1038/cddis.2012.69

70. Buerger C, Richter B, Woth K, Salgo R, Malisiewicz B, Diehl S, et al. Interleukin- $1 \beta$ interferes with epidermal homeostasis through induction of insulin resistance: implications for psoriasis pathogenesis. J Invest Dermatol. (2012) 132:2206-14. doi: 10.1038/jid.2012.123

71. Shirsath N, Mayer G, Singh TP, Wolf P. 8-methoxypsoralen plus UVA (PUVA) therapy normalizes signalling of phosphorylated component of mTOR pathway in psoriatic skin of K5.hTGF $\beta 1$ transgenic mice. Exp Dermatol. (2015) 24:889-91. doi: 10.1111/exd.12779

72. Buerger C. Epidermal mTORC1 signaling contributes to the pathogenesis of psoriasis and could serve as a therapeutic target. Front Immunol. (2018) 9:2786. doi: 10.3389/fimmu.2018.02786

73. Jones RG, Pearce EJ. MenTORing immunity: mTOR signaling in the development and function of tissue-resident immune cells. Immunity. (2017) 46:730-42. doi: 10.1016/j.immuni.2017.04.028

74. Ochaion A, Bar-Yehuda S, Cohen S, Barer F, Patoka R, Amital H, et al. The anti-inflammatory target $\mathrm{A}(3)$ adenosine receptor is over-expressed in rheumatoid arthritis, psoriasis and Crohn's disease. Cell Immunol. (2009) 258:115-22. doi: 10.1016/j.cellimm.2009.03.020

75. Reitamo S, Spuls P, Sassolas B, Lahfa M, Claudy A, Griffiths CE. Efficacy of sirolimus (rapamycin) administered concomitantly with a subtherapeutic dose of cyclosporin in the treatment of severe psoriasis: a randomized controlled trial. $\mathrm{Br} J$ Dermatol. (2001) 145:438-45. doi: 10.1046/j.1365-2133.2001.04376.x

76. Wei KC, Lai PC. Combination of everolimus and tacrolimus: a potentially effective regimen for recalcitrant psoriasis. Dermatol Ther. (2015) 28:257. doi: $10.1111 /$ dth. 12176

77. Ormerod AD, Shah SA, Copeland P, Omar G, Winfield A Treatment of psoriasis with topical sirolimus: preclinical development and a randomized, double-blind trial. $\mathrm{Br} J$ Dermatol. (2005) 152:758-64. doi: 10.1111/j.1365-2133.2005.06438.x

78. Paganelli A, Tarentini E, Benassi L, Kaleci S, Magnoni C. Mesenchymal stem cells for the treatment of psoriasis: a comprehensive review. Clin Exp Dermatol. (2020) 45:824-30. doi: 10.1111/ced.14269

79. Campanati A, Orciani M, Gorbi S, Regoli F, Di Primio R, Offidani A. Effect of biologic therapies targeting tumour necrosis factor- $\alpha$ on cutaneous mesenchymal stem cells in psoriasis. Br J Dermatol. (2012) 167:6876. doi: 10.1111/j.1365-2133.2012.10900.x

80. Chen H, Niu JW, Ning HM, Pan X, Li XB, Li Y, et al. Treatment of psoriasis with mesenchymal stem cells. Am J Med. (2016) 129:e134. doi: 10.1016/j.amjmed.2015.11.001

81. De Jesus MM, Santiago JS, Trinidad CV, See ME, Semon KR, Fernandez MO Jr, et al. Autologous adipose-derived mesenchymal stromal cells for the treatment of psoriasis vulgaris and psoriatic arthritis: a case report. Cell Transplant. (2016) 25:2063-9. doi: 10.3727/096368916X691998

82. Seetharaman R, Mahmood A, Kshatriya P, Patel D, Srivastava A. Mesenchymal stem cell conditioned media ameliorate psoriasis vulgaris: a case study. Case Rep Dermatol Med. (2019) 2019:8309103. doi: 10.1155/2019/ 8309103

83. Martins AM, Ascenso A, Ribeiro HM, Marto J. Current and future therapies for psoriasis with a focus on serotonergic drugs. Mol Neurobiol. (2020) 57:2391-419. doi: 10.1007/s12035-020-01889-3 
84. Lai R, Xian D, Xiong X, Yang L, Song J, Zhong J. Proanthocyanidins: novel treatment for psoriasis that reduces oxidative stress and modulates Th17 and Treg cells. Redox Rep. (2018) 23:130-5. doi: 10.1080/13510002.2018.1462027

85. Zhu Y, Ma Y, Zu W, Song J, Wang H, Zhong Y, et al. Identification of N-Phenyl-7H-pyrrolo[2,3-d]pyrimidin-4-amine derivatives as novel, potent, and selective NF- $\mathrm{B}$ Inducing Kinase (NIK) inhibitors for the treatment of psoriasis. J Med Chem. (2020) 63:6748-73. doi: 10.1021/acs.jmedchem.0c00055

86. Sugiura K. The genetic background of generalized pustular psoriasis: IL36RN mutations and CARD14 gain-of-function variants. J Dermatol Sci. (2014) 74:187-92. doi: 10.1016/j.jdermsci.2014.02.006

87. Papp KA, Menter A, Strober B, Langley RG, Buonanno M, Wolk $R$, et al. Efficacy and safety of tofacitinib, an oral Janus kinase inhibitor, in the treatment of psoriasis: a phase $2 b$ randomized placebo-controlled dose-ranging study. $\mathrm{Br} J$ Dermatol. (2012) 167:668-77. doi: 10.1111/j.1365-2133.2012.11168.x

88. Bachelez H, van de Kerkhof PC, Strohal R, Kubanov A, Valenzuela F, Lee JH, et al. Tofacitinib versus etanercept or placebo in moderate-to-severe chronic plaque psoriasis: a phase 3 randomised non-inferiority trial. Lancet. (2015) 386:552-61. doi: 10.1016/S0140-6736(14)62113-9

89. Leonardi CL, Kimball AB, Papp KA, Yeilding N, Guzzo C, Wang Y, et al. Efficacy and safety of ustekinumab, a human interleukin-12/23 monoclonal antibody, in patients with psoriasis: 76-week results from a randomised, double-blind, placebo-controlled trial (PHOENIX 1). Lancet. (2008) 371:1665-74. doi: 10.1016/S0140-6736(08)60725-4

90. Papp KA, Langley RG, Lebwohl M, Krueger GG, Szapary P, Yeilding $\mathrm{N}$, et al. Efficacy and safety of ustekinumab, a human interleukin-12/23 monoclonal antibody, in patients with psoriasis: 52-week results from a randomised, double-blind, placebo-controlled trial (PHOENIX 2). Lancet. (2008) 371:1675-84. doi: 10.1016/S0140-6736(08)60726-6

91. Griffiths CE, Strober BE, van de Kerkhof P, Ho V, Fidelus-Gort R, Yeilding $\mathrm{N}$, et al. Comparison of ustekinumab and etanercept for moderate-to-severe psoriasis. N Engl J Med. (2010) 362:118-28. doi: 10.1056/NEJMoa0810652

92. Blauvelt A, Papp KA, Griffiths CE, Randazzo B, Wasfi Y, Shen YK, et al. Efficacy and safety of guselkumab, an anti-interleukin-23 monoclonal antibody, compared with adalimumab for the continuous treatment of patients with moderate to severe psoriasis: results from the phase III, doubleblinded, placebo- and active comparator-controlled VOYAGE 1 trial. J Am Acad Dermatol. (2017) 76:405-17. doi: 10.1016/j.jaad.2016.11.041
93. Reich K, Armstrong AW, Foley P, Song M, Wasfi Y, Randazzo $\mathrm{B}$, et al. Efficacy and safety of guselkumab, an anti-interleukin-23 monoclonal antibody, compared with adalimumab for the treatment of patients with moderate to severe psoriasis with randomized withdrawal and retreatment: Results from the phase III, doubleblind, placebo- and active comparator-controlled VOYAGE 2 trial J Am Acad Dermatol. (2017) 76:418-31. doi: 10.1016/j.jaad.2016. 11.042

94. Reich K, Papp KA, Blauvelt A, Tyring SK, Sinclair R, Thaçi D, et al. Tildrakizumab versus placebo or etanercept for chronic plaque psoriasis (reSURFACE 1 and reSURFACE 2): results from two randomised controlled, phase 3 trials. Lancet. (2017) 390:276-88. doi: 10.1016/S0140-6736(17)31279-5

95. Gordon KB, Strober B, Lebwohl M, Augustin M, Blauvelt A, Poulin Y, et al. Efficacy and safety of risankizumab in moderate-to-severe plaque psoriasis (UltIMMa-1 and UltIMMa-2): results from two double-blind, randomised, placebo-controlled and ustekinumab-controlled phase 3 trials. Lancet. (2018) 392:650-61. doi: 10.1016/S0140-6736(18)31713-6

96. Bachelez H, Choon SE, Marrakchi S, Burden AD, Tsai TF, Morita A, et al. Trial of spesolimab for generalized pustular psoriasis. N Engl J Med. (2021) 385:2431-40. doi: 10.1056/NEJMoa2111563

Conflict of Interest: The authors declare that the research was conducted in the absence of any commercial or financial relationships that could be construed as a potential conflict of interest.

Publisher's Note: All claims expressed in this article are solely those of the authors and do not necessarily represent those of their affiliated organizations, or those of the publisher, the editors and the reviewers. Any product that may be evaluated in this article, or claim that may be made by its manufacturer, is not guaranteed or endorsed by the publisher.

Copyright (c) 2022 Thakur and Mahajan. This is an open-access article distributed under the terms of the Creative Commons Attribution License (CC BY). The use, distribution or reproduction in other forums is permitted, provided the original author(s) and the copyright owner(s) are credited and that the original publication in this journal is cited, in accordance with accepted academic practice. No use, distribution or reproduction is permitted which does not comply with these terms. 